\title{
Tracheocutaneous Fistula in Children Following Tracheostomy Decannulation: Can Imaging Guide the Management Algorithm?
}

\author{
Pratibha Bhatia ${ }^{1}$ Anmol Bhatia ${ }^{1}$ Akshay Kumar Saxena ${ }^{1} \cdot$ Joseph L Mathew ${ }^{2}$. Jaimanti Bakshi ${ }^{3}$. \\ Kushaljit Singh Sodhi ${ }^{1}$
}

Received: 24 April 2021 / Accepted: 19 July 2021 / Published online: 17 August 2021

(c) Dr. K C Chaudhuri Foundation 2021

To the Editor: Persistent tracheocutaneous fistula (TCF) following decannulation is encountered in approximately onefourth of pediatric patients with history of long-term cannulation $[1,2]$. Limited data is available regarding the role of imaging in TCF. Herein, we present a child with TCF, with emphasis on the radiological findings and their importance in deciding the further management.

A 6-y-old girl child was tracheostomized for 8 mo following complicated meningitis. On decannulation, the patient developed TCF. Two years later, even though there was visible reduction in the size of external fistulous opening, the parents were concerned about the persistent discharge and difficulty in speech, apart from the cosmetic issues. Computed tomography (CT) scan showed a linear fistulous tract ( $<5 \mathrm{~mm}$ in size), measuring approximately $6 \mathrm{~mm}$ in length, arising from the anterior wall of trachea reaching up to the skin surface. Rest of the airway was unremarkable. Patient underwent surgical closure of TCF and started on speech therapy.

Management of TCF is still not uniform with no definite guidelines [3]. CT and magnetic resonance imaging (MRI) play an important role in demonstration of various airway, mediastinal and lung pathologies in children [4]. Imaging not only confirms the presence of TCF but also helps in devising further treatment strategy. Precise demonstration of the dimensions and location of fistula plays a significant role in deciding further treatment plan. Patients with fistula

Kushaljit Singh Sodhi

sodhiks@gmail.com

1 Department of Radiodiagnosis and Imaging, Postgraduate Institute of Medical Education and Research, Chandigarh 160012, India

2 Department of Pediatrics, Postgraduate Institute of Medical Education and Research, Chandigarh, India

3 Department of Otorhinolaryngology, Postgraduate Institute of Medical Education and Research, Chandigarh, India size $<5 \mathrm{~mm}$ may be simply observed for spontaneous resolution [3]. Presence of airway stenosis, web, tracheal ring, and tracheomalacia which require modification of surgical technique can be well assessed on CT scan. Virtual bronchoscopy not only allows intraluminal assessment but can also negotiate across the stenotic site, which is difficult via fibreoptic bronchoscopy. Newer studies have suggested role of MRI in imaging large airways in children considering the risk of radiation exposure and may be inculcated as a routine in future practice.

\section{Declarations}

Conflict of Interest None.

\section{References}

1. Cheng J, Setabutr D. Tracheocutaneous fistula closure in children. Int J Pediatr Otorhinolaryngol. 2016;89:107-11.

2. Rajagopalan R, Parambil R, Sappavoo K, et al. Persistent tracheocutaneous fistula: a case report. Respir Case Reports. 2018;7:140-4.

3. Kao C-N, Liu Y-W, Chang P-C, et al. Decision algorithm and surgical strategies for managing tracheocutaneous fistula. J Thorac Dis. 2020;12:457-65.

4. Sodhi KS, Khandelwal N, Saxena AK, et al. Rapid lung MRI in children with pulmonary infections: Time to change our diagnostic algorithms. J Magn Reson Imaging. 2016;43:1196-206.

Publisher's Note Springer Nature remains neutral with regard to jurisdictional claims in published maps and institutional affiliations. 